\title{
An Efficient and Simple Method for Modelling Solar Cells
}

\author{
Ali Kareem Abdulrazzaq1,2*, György Bognár ${ }^{1}$, Balázs Plesz ${ }^{1}$ \\ ${ }^{1}$ Department of Electron Devices, Faculty of Electrical Engineering and Informatics, Budapest University of Technology and \\ Economics, Magyar tudósok körútja 2, H-1117 Budapest, Hungary \\ 2 Department of Electrical and Electronics Engineering, Thi-Qar University, 64001 Nassiriya, P. O. B. 0535, Iraq \\ * Corresponding author, e-mail: kareem@eet.bme.hu
}

Received: 16 May 2019, Accepted: 27 August 2019, Published online: 10 October 2019

\begin{abstract}
Accurate and reliable PV device modelling is a fundamental tool to optimize system performance. The regular operation of the PV system in an outdoor condition implies the need for a model that relate the environmental effects. This paper presents a new, simple, and efficient method for constructing the $I-V$ characteristics for a PV cell incorporating the environmental conditions. The proposed model is based on the idea of dividing the voltage axis of the solar cell $I-V$ characteristics using a fixed time interval. For each voltage interval point, a two-dimensional current matrix is calculated, corresponding different irradiance conditions and temperatures. Polynomial surface fitting is used to build sub-models for each voltage interval. The current two-dimensional matrix, for each point, is calculated by two different methods. The first method is based on linear interpolation of the measured $I-V$ curves. The second method uses pre-extracted five parameters for the well known single diode model at a wide range of environmental conditions. The developed modelling technique provides accurate results compared with the measured data for a mono-crystalline solar cell.
\end{abstract}

Keywords

solar cell, I-V characteristics, single diode model

\section{Introduction}

The accurate power prediction under various environmental conditions is a crucial issue for the PV system power investment. Developing countries are facing severe challenges in selecting the proper PV modules for having efficient power production to cope with various environmental conditions. A reliable modelling tool is essential to optimize system performance and cost-effectiveness [1-3]. The PV module's datasheet information measured at standard testing conditions (STC) (i.e. irradiance $=1000 \mathrm{~W} / \mathrm{m}^{2}$, temperature $=25^{\circ} \mathrm{C}$, and AM1.5) is not sufficient to build accurate and reliable system models that are able to take into account the changing operating conditions like different irradiance conditions and varying temperatures [4]. Researchers are using various approaches for describing the current-voltage characteristics ( $I-V$ curves), each with different theoretical and physical basement and different level of accuracy of the final results. Some methods are entirely empirical [5-7]. These models are not based on theoretical descriptions. The primary goal of these approaches is to have an explicit mathematical description of the current in dependence on the voltage and other elementary environmental factors. Such methods that do not include technological or structural parameters of the device have the drawback of limitation in exploring the device behavior. An advantage of these methods of modelling is not losing accuracy due to relying on imprecise physical definitions.

In [5] the authors proposed a model for the $I-V$ characteristics given as

$$
I=\frac{V_{O C}-V}{A+B V^{2}-C V}
$$

where the coefficient $A$ represents the ratio of the open circuit voltage $\left(V_{O C}\right)$ to the short circuit current $\left(I_{S C}\right), B$ and $C$ are calculated from two selected points of the measured $I-V$ curve. An extension for this work has been presented in [6] to obtain a closed-form solution for transient voltage and current when a solar device is loaded with $R-L$ or $R-C$ loads. Their model is given as

$$
I=\sqrt{K_{11} V^{2}+K_{2} V+K_{31}},
$$

where $K_{11}, K_{2}$ and $K_{31}$ are obtained by satisfying the three characteristics points: the open circuit, maximum power and short circuit of the PV device. The research work presented in [7] introduces a characteristics model that takes 
into consideration the temperature of the panel and the effect of the irradiance. Their proposed model is given as

$$
I(V)=\alpha \cdot I_{\max } \cdot \tau_{i}\left(1-e^{\left(\frac{V}{b \cdot(\gamma \cdot \alpha+1-\gamma) \cdot\left(V_{\max }+\tau_{v}\right)}-\frac{1}{b}\right)}\right),
$$

where $\alpha$ is the percentage of the effective light intensity, $\tau_{i}$ is the rate of change for the current, $\tau_{v}$ is the rate of change for the voltage, $I_{\max }$ is the ideal maximum current (when $V=-\infty$ at STC), $b$ is the characteristics $I-V$ constant, $\gamma$ is the shading linear factor, $V_{\max }$ is the open circuit voltage at $25^{\circ} \mathrm{C}$ and $1000 \mathrm{~W} / \mathrm{m}^{2}$.

The other main modelling approach is based on a theoretical foundation that describes the operation of the PV device considering the technological parameters. Using deep theoretical knowledge provide the ability for predicting the effects of changes in materials, technology or structural parameters. The difficulty in applying such an approach is the availability of the technological and structural parameters which are required to build the model. The work presented in Vaillon et al. [8] is considered as an example of the theoretical model, in which, the authors proposed a physical and numerical solar cell model as a function of the irradiance and temperature. Their model considers the solar cell as a single $p$ - $n$ junction as a simplified case to develop a coupled heat transfer - photoelectric phenomena comprehensive model.

Approaches incorporating both empirical and theoretical modelling concepts are considered semi-empirical models. Thereby, combining the advantages of the behavioral description of the $p-n$ junction that includes physical parameters and also based on measurements. This approach is implemented by one of the two following modelling techniques: single diode model (SDM) and the double diode model (DDM).

Fig. 1 shows the SDM model in which a single diode is imitating both the charge carries diffusion and the recombination in the depletion region. In the DDM model, these two physical phenomena are modelled with a separate diodes, as shown in Fig. 2.

Both models are implicit and non-linear. Although the DDM model is considered to be more accurate,

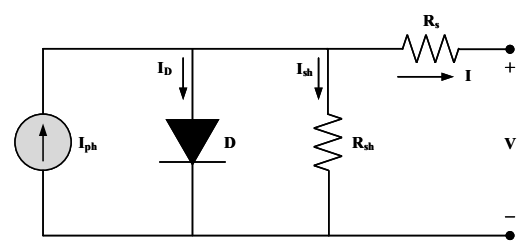

Fig. 1 The single diode model equivalent circuit of a PV cell.

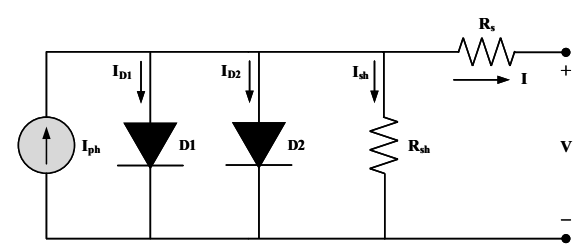

Fig. 2 The double diode model equivalent circuit of a PV cell.

more characteristics parameters have to be determined. The SDM is widely used by the researchers for modelling PV cells because it represents a good compromise between complicity and accuracy due to less number of parameters that have to be calculated as well as the excellent level of accuracy [4, 9-15]. The main physical reason for that is the minor effect of the charge carriers recombination near the depletion region at a high operating voltages that are typical for solar cells. On the other hand at lower voltages the photocurrent dominates and once again, suppresses the recombination current of the diode.

The mathematical expression of the SDM is given as

$I=I_{p h}-I_{o}\left(e^{\frac{\left(V+I R_{s}\right)}{n V_{T}}}-1\right)-\frac{V+I R_{s}}{R_{s h}}$

where $\left(I_{p h}\right)$ is the photogenerated current, $\left(I_{o}\right)$ is the reverse saturation current, $(n)$ is the ideality factor of the PV cell, $\left(R_{s}\right)$ is the cell series resistance, and $\left(R_{s h}\right)$ is the shunt resistance.

In this paper, a new solar cell modelling technique is proposed to reproduce the $I-V$ characteristic curve at any irradiance condition and temperature. The model is based on the consent of dividing the voltage axis with a fixed interval. The voltage interval points track the solar cell output current values corresponding to different environmental condition. For each voltage interval point, it is required to record the currents under a wide range of measured $I-V$ curves to produce a two-dimensional current matrix. A sub-model is constructed for each point using a polynomial surface fitting. The top-level model that provides an $I-V$ curve at any given environmental condition is based on the combination of all sub-modules, by evaluating them and calculating the current for the corresponding voltage interval point.

\section{Theory}

The work presented in this paper is based on tracking the current values corresponding to fixed voltage interval points. Each one of these voltage intervals will be subject for investigating the irradiance and temperature dependence of the output current. This concept has been implemented by utilizing two different methods to determine 
the currents values corresponding to a specific voltage interval under different irradiance conditions and temperatures $I_{i}(G, T)$, where $i$ is the voltage interval points index. As a result, we will get a two-dimensional matrix of current values for each voltage interval in dependence of the temperature and the irradiance, representing the first part of building the model. The second part includes fitting a polynomial surface to create a sub-model for each voltage interval point. The following two subsections show more details about building the model.

\subsection{Construction of the two-dimensional current matrix for each voltage interval point}

Linear interpolation method (LIM) is the first technique for calculating the current matrix $I_{i}(G, T)$. The available measured $I-V$ curves include data points where the voltage steps between the measurement points are different from one curve to another. This method aims to reproduce the measured $I-V$ curve, such that, the new interpolated data is selected to be always at the same voltage interval. Hence, a new $I-V$ data set is generated corresponding each measured curve under different environmental conditions, from which, we shall construct the $I_{i}(G, T)$ matrix.

The second method for calculating the current matrix is based on the pre-extracted five parameters of the SDM (we use the abbreviation SDMBM for this method) for each measured curve under the environmental investigation range. Based on the SDM mathematical representation, the five parameters are the photogenerated current $\left(I_{p h}\right)$, reverse saturation current $\left(I_{o}\right)$, the ideality factor $(n)$, series resistance $\left(R_{s}\right)$, and shunt resistance $(R s h)$. For this purpose, we use the results of our ongoing research work which was focusing on extracting the five characteristics parameters for the SDM solar cell/module equivalent circuit. In which, the least square method is used as an error minimization technique for fitting the non-linear transcendental model equation of the solar panel to the measured $I-V$ characteristics. Newton Raphson method is applied to solve the system of five non-linear equations which represent the error of each parameter.

For each voltage interval point, the current values will be calculated using the extracted five parameters corresponding to each input $I-V$ curve. The used formula for calculating the currents is the explicit form of the SDM based on the Lambert $W$-function given as [16-18]:

$$
\begin{aligned}
I & =\frac{R_{s h}\left(I_{p h}+I_{o}\right)}{R_{s h}+R_{s}}-\frac{n V_{T}}{R_{s}} W\left(\frac{R_{s} I_{o}}{n V_{T}} \frac{R_{s h}}{R_{s h}+R_{s}} e^{\frac{R_{s h}\left(V+R_{s h}\left(I_{p h}+I_{o}\right)\right)}{n V_{T}\left(R_{s h}+T_{s}\right)}}\right) \\
& -\frac{V}{R_{s h}+R_{s}},
\end{aligned}
$$

where $W$ is the principal branch of the Lambert $W$-function.

\subsection{Surface fitting for constructing the model}

Using both methods from Section 2.1, considering the availability of measurement at different temperatures and irradiance conditions, a two-dimensional matrix of current values will be calculated for each voltage interval point. A polynomial surface function is required to be fitted to the current matrix data to generate a sub-model corresponding to each voltage interval point.

For this purpose we are using a fifth order polynomial surface function. Hence, there are 21 coefficients for each voltage interval points. Although, there is a large number of coefficients; however, calculating the coefficients has to be done only once. Fig. 3 (a)-(c) shows an example of the fitted surface for the current values for the voltage interval points $0.1 \mathrm{~V}, 0.2 \mathrm{~V}$, and $0.3 \mathrm{~V}$, respectively, using the second method (based on the SDM pre-extracted parameters).

The mathematical representation of the sub-models resulting from the fitted function is given as

$$
\begin{aligned}
I_{\text {OUT }}^{i}= & \sum_{k=1}^{3} B_{k}^{i} \times T^{k-1} \times G^{k-1}+\sum_{l=2}^{4} B_{l+2}^{i} \times T \times G^{l} \\
& +\sum_{m=2}^{4} B_{m+5}^{i} \times T^{m} \times G+\sum_{n=1}^{5} B_{n+9}^{i} \times T^{n} \\
& +\sum_{p=1}^{5} B_{p+14}^{i} \times G^{p}+\sum_{q=2}^{3} B_{q+18}^{i} \times T^{5-q} \times G^{q}
\end{aligned}
$$

where $I_{i}^{\text {out }}$ is the calculated current value corresponding to a specific voltage interval point $(i), B_{i}^{1}-B_{i}^{21}$ are the fitting coefficients corresponding to the surface function of the current at the same voltage interval point in dependence on the irradiation and the temperature. $T$ and $G$ are the targeted temperature and irradiance conditions, respectively, at which a new $I-V$ curve is required to be constructed.

The final step is to calculate the current corresponding to each voltage interval point using the corresponding coefficients as a function of the targeted $T$ and $G$, and by considering only the $I-V$ points in the first power quadrant. 


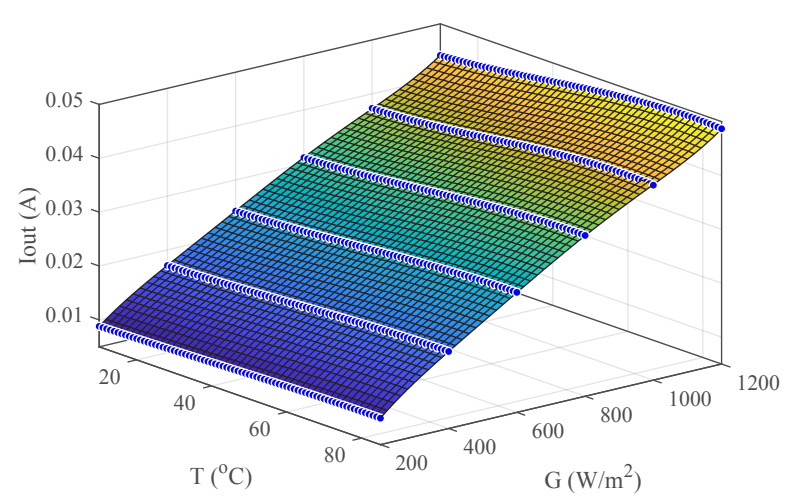

(a)

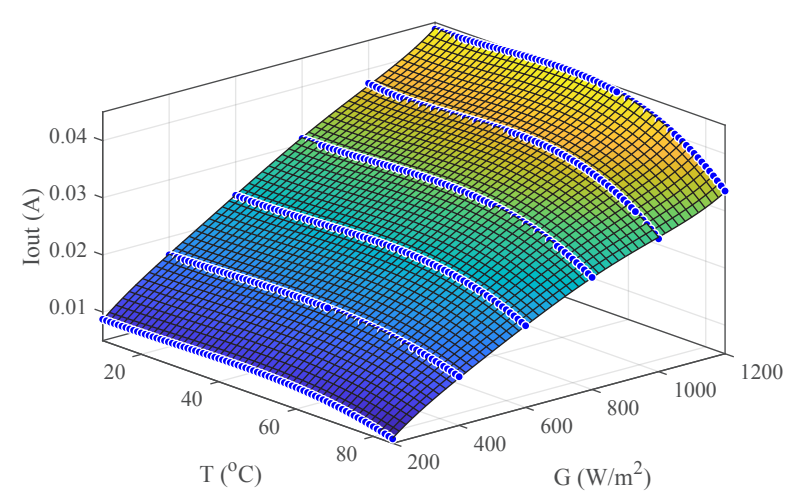

(b)

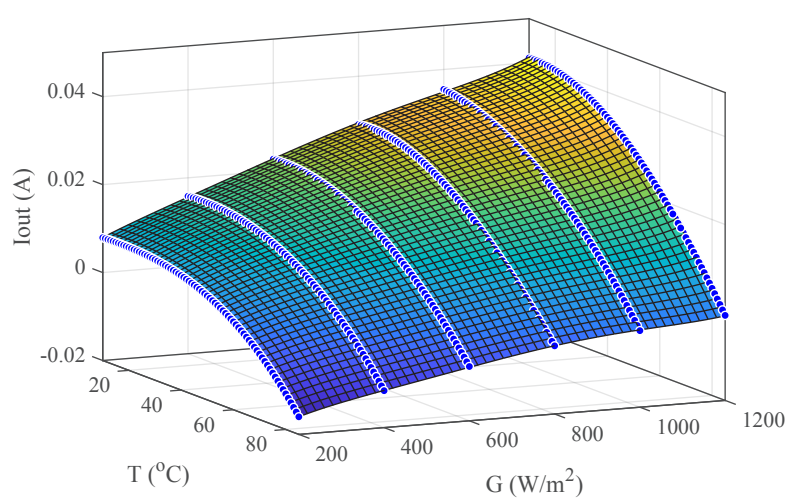

(c)

Fig. 3 Surface fitting for the current values corresponding to a single voltage interval point on the axis under different environmental conditions. (a) $V=0.1 \mathrm{~V}$. (b) $V=0.2 \mathrm{~V}$. (c) $V=0.3 \mathrm{~V}$.

\section{Results and discussion}

For testing and validating the proposed model we use $I-V$ curves measurement under irradiance range of $200 \mathrm{~W} / \mathrm{m}^{2}-1200 \mathrm{~W} / \mathrm{m}^{2}$ and temperature range of $10{ }^{\circ} \mathrm{C}-$ $85{ }^{\circ} \mathrm{C}$, for a mono-crystalline cell from previous laboratory measurements [17]. For this PV sample, the maximum voltage that could be realized as an output under the considered environmental ranges is $0.5 \mathrm{~V}$; therefore, 50 points starting from $0.01 \mathrm{~V}$ up to $0.5 \mathrm{~V}$ (voltage interval is $0.01 \mathrm{~V}$ ) has been selected to construct the model.
For $I(G, T)$ matrix using both proposed method, Eq. (6) is used constructing a new $I-V$ curve at any selected irradiance $(G)$ and temperature $(T)$, by calculating the current value corresponding to each voltage interval.

Fig. 4 (a), (b) shows the constructed I-V curves of the PV sample at different temperatures and irradiance conditions, respectively, for the linear interpolation method (LIM). While Fig. 5 (a), (b) shows the results when using the SDM pre-extracted parameters based model (SDMBM).

Evaluating the performance of the model presented in this work and quantifying the error is performed by calculating the error at the maximum power point $(\delta)$, and the mean absolute percentage error (MAPE).

Tables 1 and 2 show the evaluating parameters for the proposed model based on both $I(G, T)$ calculation methods, for different irradiance conditions and different temperatures, respectively. Table 1 shows the result of different irradiance conditions at $20{ }^{\circ} \mathrm{C}$ temperature. Table 2 shows the result of different temperatures at $1000 \mathrm{~W} / \mathrm{m}^{2}$ irradiance.

Table 3 shows the performance monitoring parameters which are calculated by comparing 456 constructed $I-V$ curves with the measured characteristics

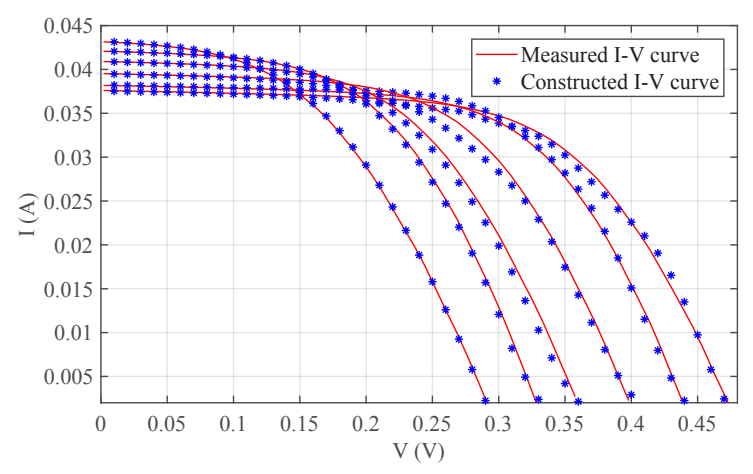

(a)

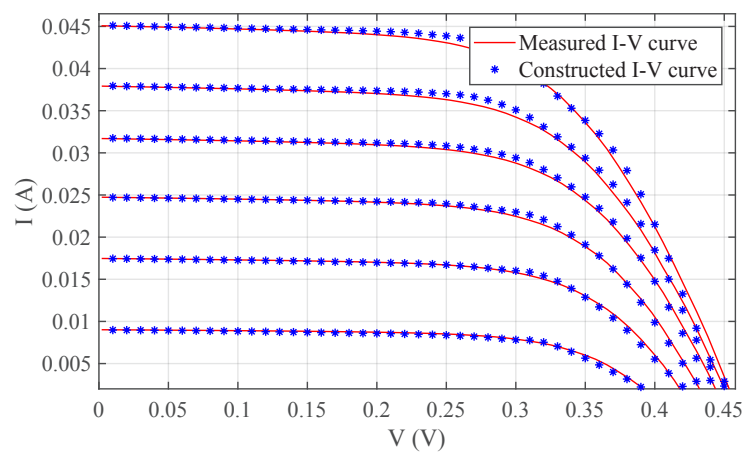

(b)

Fig. 4 New constructed I-V curves based on the LIM together with the measured data (a) At $1000 \mathrm{~W} / \mathrm{m}^{2}$ and different temperatures $(10-85){ }^{\circ} \mathrm{C}$.

(b) At $20{ }^{\circ} \mathrm{C}$ and different irradiance conditions (200-1200) W/m². 


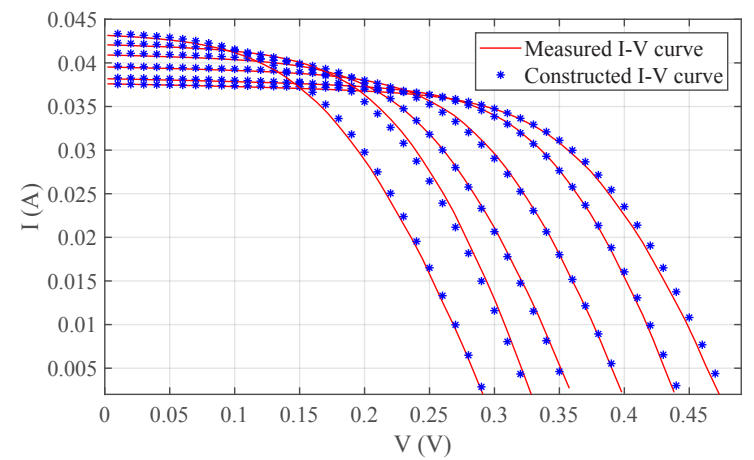

(a)

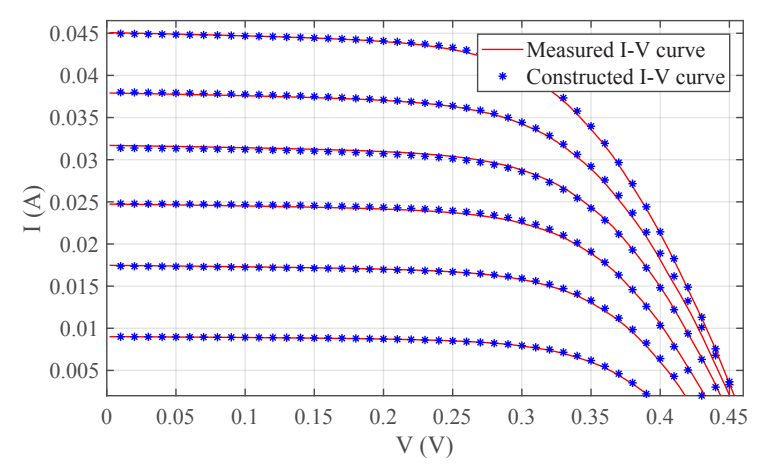

(b)

Fig. 5 New constructed I-V curves based on the SDMBM together with the measured data. (a) At $1000 \mathrm{~W} / \mathrm{m}^{2}$ and different temperatures

(10-85) ${ }^{\circ} \mathrm{C}$. (b) At $20{ }^{\circ} \mathrm{C}$ and different irradiance conditions $(200-1200) \mathrm{W} / \mathrm{m}^{2}$.

Table 1 Model evaluation at different irradiance conditions for both $I(G, T)$ calculation methods.

\begin{tabular}{lccc}
\hline Method & $G 2\left(\mathrm{~W} / \mathrm{m}^{2}\right)$ & $\delta \%$ & MAPE $\%$ \\
\hline 200 & 1.87 & 1.37 \\
LIM & 400 & 2.78 & 0.14 \\
& 600 & 2.82 & 5.44 \\
& 800 & 1.80 & 0.26 \\
& 1000 & 1.38 & 0.78 \\
& 1200 & 0.57 & 0.66 \\
\hline \multirow{3}{*}{ SDMBM } & 200 & 1.10 & 0.47 \\
& 400 & 1.37 & 0.96 \\
& 600 & 1.32 & 0.19 \\
& 800 & 0.66 & 0.85 \\
& 1000 & 0.60 & 2.13 \\
& 1200 & 0.99 & 0.49 \\
\hline
\end{tabular}

at the corresponding environmental conditions, using the proposed model based on both presented current calculation methods.

The presented validation results reveal the applicability under a wide environmental range using both of the introduced methods for calculating the current matrices values.
Table 2 Model evaluation at different temperatures for both $I(G, T)$ calculation methods.

\begin{tabular}{lccc}
\hline Method & $T 2\left({ }^{\circ} \mathrm{C}\right)$ & $\delta \%$ & MAPE $\%$ \\
\hline \multirow{4}{*}{ LIM } & 10 & 1.36 & 0.66 \\
& 25 & 0.97 & 0.13 \\
& 40 & 5.08 & 1.68 \\
& 55 & 2.41 & 3.31 \\
& 70 & 1.00 & 5.15 \\
SDMBM & 85 & 0.78 & 0.12 \\
& 10 & 0.53 & 3.97 \\
& 25 & 0.54 & 1.50 \\
& 40 & 1.75 & 0.24 \\
& 55 & 0.20 & 0.92 \\
& 70 & 2.67 & 3.40 \\
& 85 & 2.71 & 2.48 \\
\hline
\end{tabular}

Table 3 Evaluation of the proposed model using 456 curves at different environmental conditions.

\begin{tabular}{lcccc}
\hline Method & Parameter & $\begin{array}{c}\text { Average } \\
\text { value }\end{array}$ & $\begin{array}{c}\text { Worst } \\
\text { recorded } \\
\text { value }\end{array}$ & $\begin{array}{c}\text { Percentage of } \\
\text { curves with } \\
\text { error value } \\
>5 \%\end{array}$ \\
\hline \multirow{2}{*}{ LIM } & $\delta$ & $1.69 \%$ & $7.77 \%$ & $3.2 \%$ \\
\multirow{2}{*}{ SDMBM } & MAPE & $1.86 \%$ & $5.93 \%$ & $4.8 \%$ \\
& MAPE & $2.11 \%$ & $5.69 \%$ & $2.1 \%$ \\
\hline
\end{tabular}

However, from the Figs. 4 and 5 and from the Tables 1, 2 , and 3 we can conclude a slightly better performance in case of using the SDM based method for constructing the current matrices values.

As shown in Table 3, the average value of the MAPE is below $2 \%$ using both methods for extracting the current matrices. The average error at the maximum power point is $1.69 \%$ when using LIM, while it is $2.11 \%$ with the SDMBM. However, this should not be considered as a performance advantage for the LIM, because both method inherent very low error value and these numbers are very close to each other. The last column in Table 3, shows the percentages that indicate how many modelled curves out of the 456 include errors above $5 \%$ for both error evaluation parameters. From this part of Table 3, it can be seen that SDMBM give better result, compared with LIM, for both error parameters values, where no MAPE was recorded above $5 \%$ and only $2.1 \%$ of the tested curve got $\delta$ values between $5 \%$ and $5.69 \%$ as maximum.

Reducing the amount of data measurement is considered as a practical solution in PV modelling. Table 4 shows the result of reproducing $456 I-V$ curves using a decreasing number of the measured $I-V$ curves. The reduction of 
Table 4 Model evaluation using different numbers of $I$ - $V$ curves within the same range of the environmental conditions.

\begin{tabular}{lccccc}
\hline & \multicolumn{5}{c}{ Average value } \\
No. of I-V curves & 456 & 288 & 152 & 90 & 57 \\
\hline$\delta$ & $1.69 \%$ & $2.31 \%$ & $5.17 \%$ & $6.75 \%$ & $8.95 \%$ \\
MAPE & $1.86 \%$ & $3.12 \%$ & $6.60 \%$ & $8.38 \%$ & $10.62 \%$ \\
\hline
\end{tabular}

the measurement is carried out by considering a different interval of the temperature $\left(2^{\circ} \mathrm{C}, 3^{\circ} \mathrm{C}, 5^{\circ} \mathrm{C}\right.$, and $\left.8^{\circ} \mathrm{C}\right)$; hence, we investigate constructing the model using 288, 152, 90 , and $57 \mathrm{I}-\mathrm{V}$ curves, respectively. The table shows an increasing value of the error quantifying parameters with decreasing the number of measurements used to build the model. However, with reducing the $I-V$ curves to the half, we still see average error value below $5 \%$ for both parameters.

\section{Conclusion}

In this paper, a new, simple, and accurate model for constructing the PV cell $I-V$ characteristics were developed and presented. The model concept is based on considering the interval axis as fixed interval points for tracking the current value changes under different irradiance conditions and different temperatures. The model is constructed in two steps. The first step is to calculate the current values under different environmental conditions $I(G, T)$ for each voltage interval point. This concept was

\section{References}

[1] Cubas, J., Pindado, S., De Manuel, C. "Explicit Expressions for Solar Panel Equivalent Circuit Parameters Based on Analytical Formulation and the Lambert W-Function", Energies, 7(7), pp. 4098-4115, 2014. https://doi.org/10.3390/en7074098

[2] Cuce, E., Cuce, P. M., Karakas, I. H., Bali, T. "An accurate model for photovoltaic (PV) modules to determine electrical characteristics and thermodynamic performance parameters", Energy Conversion and Management, 146, pp. 205-216, 2017. https://doi.org/10.1016/j.enconman.2017.05.022

[3] International Atomic Energy Agency "Application of Reliability Centred Maintenance to Optimize Operation and Maintenance in Nuclear Power Plants", IAEA, Vienna, Austria, Rep. IAEATECDOC-1590, 2007.

[4] Peñaranda Chenche, L. E., Hernandez Mendoza, O. S., Bandarra Filho, E. P. "Comparison of four methods for parameter estimation of mono- and multi-junction photovoltaic devices using experimental data", Renewable and Sustainable Energy Reviews, 81(2), pp. 2823-2838, 2018.

https://doi.org/10.1016/j.rser.2017.06.089 implemented by two different methods, namely, the linear interpolation and the other method is based on using the pre-extracted five parameters of SDM. The second step is performed by fitting a polynomial surface to the resulting two-dimensional current matrix and creating a sub-model for each voltage interval point. The model was verified using a mono-crystalline solar cell with 456 measured curves at a wide range of environmental parameters. Evaluating the performance of the proposed model was by calculating the error at the maximum power point, and MAPE. The overall average MAPE was below $2 \%$. The model based on the LIM shows the lowest error at the maximum power point with an average value of $1.69 \%$. The results based on SDMBE provide modelling results with no MAPE above $5 \%$. From this one can conclude that the constructed model is efficient under the different environmental condition, that proves its applicability, for both current calculation methods without a significant advantage of one of them over the other. A notable advantage of this model is that it mitigates the possible $I-V$ measurement errors since it relies on surface fitting. However, accurate modelling requires a large number of measured $I-V$ curves at a wide range of irradiance conditions and temperatures. The proposed modelling method best performance is within the ranges of the measurements environmental conditions used for constructing the model.

[5] Akbaba, M., Alattawi, M. A. A. "A new model for $I-V$ characteristic of solar cell generators and its applications", Solar Energy Materials and Solar Cells, 37(2), pp. 123-132, 1995. https://doi.org/10.1016/0927-0248(94)00201-0

[6] Akbaba, M. "Performance analysis of solar cell arrays loaded with passive loads", Applied Energy, 52(2-3), pp. 209-218, 1995. https://doi.org/10.1016/0306-2619(95)00037-S

[7] Ortiz-Rivera, E. I., Peng, F. Z. "Analytical Model for a Photovoltaic Module using the Electrical Characteristics provided by the Manufacturer Data Sheet", In: IEEE $36^{\text {th }}$ Power Electronics Specialists Conference, Recife, Brazil, 2005, pp. 2087-2091. https://doi.org/10.1109/PESC.2005.1581920

[8] Vaillon, R., Robin, L., Muresan, C., Ménézo, C. "Modeling of coupled spectral radiation, thermal and carrier transport in a silicon photovoltaic cell", International Journal of Heat and Mass Transfer, 49(23-24), pp. 4454-4468, 2006. https://doi.org/10.1016/j.ijheatmasstransfer.2006.05.014

[9] Rhouma, M. B. H., Gastli, A., Ben Brahim, L., Touati, F., Benammar, M. "A simple method for extracting the parameters of the PV cell single-diode model", Renewable Energy, 113, pp. 885-894, 2017. https://doi.org/10.1016/j.renene.2017.06.064 
[10] Di Piazza, M. C., Luna, M., Petrone, G., Spagnuolo, G. "Translation of the Single-Diode PV Model Parameters Identified by Using Explicit Formulas", IEEE Journal of Photovoltaics, 7(4), pp. 1009-1016, 2017. https://doi.org/10.1109/JPHOTOV.2017.2699321

[11] Ayodele, T. R., Ogunjuyigbe, A. S. O., Ekoh, E. E. "Evaluation of numerical algorithms used in extracting the parameters of a single-diode photovoltaic model", Sustainable Energy Technologies and Assessments, 13, pp. 51-59, 2016.

https://doi.org/10.1016/j.seta.2015.11.003

[12] Toledo, F. J., Blanes, J. M. "Analytical and quasi-explicit four arbitrary point method for extraction of solar cell single-diode model parameters", Renewable Energy, 92, pp. 346-356, 2016. https://doi.org/10.1016/j.renene.2016.02.012

[13] Yoon, Y., Geem, Z. W. "Parameter Optimization of Single-Diode Model of Photovoltaic Cell Using Memetic Algorithm", International Journal of Photoenergy, 2015, article ID: 963562, 2015. https://doi.org/10.1155/2015/963562

[14] Ghani, F., Rosengarten, G., Duke, M., Carson, J. K. "The numerical calculation of single-diode solar-cell modelling parameters", Renewable Energy, 72, pp. 105-112, 2014.

https://doi.org/10.1016/j.renene.2014.06.035
[15] Şentürk, A. "New method for computing single diode model parameters of photovoltaic modules", Renewable Energy, 128(A), pp. 30-36, 2018.

https://doi.org/10.1016/j.renene.2018.05.065

[16] Jain, A., Kapoor, A. "Exact analytical solutions of the parameters of real solar cells using Lambert W-function", Solar Energy Materials and Solar Cells, 81(2), pp. 269-277, 2004.

https://doi.org/10.1016/j.solmat.2003.11.018

[17] Abdulrazzaq, A. K. "Electro-Thermal Modeling of Photovoltaic (PV) Systems", In: Keresztes, G., Szabó, Cs. (eds.) Spring Wind 2018, vol. 3, Association of Hungarian PhD and DLA students, Budapest, Hungary, 2018, pp. 17-29. https://doi.org/10.23715/TSZ.2018.3

[18] Plesz, B. "Investigation of the Temperature Dependence of Thin Photoactive Layer Crystalline Silicon Solar Cells", PhD Thesis, Budapest University of Technology and Economics, 2016. [online] Available at: https://repozitorium.omikk.bme.hu/bitstream/ handle $/ 10890 / 1523 /$ tezis_eng.pdf? sequence $=4 \&$ is Allowed $=y$ [Accessed: 24 April 2019] 